\title{
EVALI versus MIS-C, one more overlapping diagnosis to consider
}

\author{
Diego Cruz-Vidal ${ }^{1}$, Eric Mull ${ }^{1}$, Jeannette Taveras $^{1}$, Richard Shell ${ }^{2}$, William Hunt ${ }^{1}$, Brian \\ Fowler $^{1}$, Rebecca Wallihan ${ }^{1}$, and Guliz Erdem ${ }^{1}$ \\ ${ }^{1}$ Nationwide Children's Hospital \\ ${ }^{2}$ Nationwide Children's Hospital
}

November 12, 2020

\begin{abstract}
We describe six teenagers presenting with fever and severe abdominal symptoms admitted with concerns for multisystem inflammatory syndrome in children (MIS-C). Laboratory evaluation revealed elevated markers of inflammation, lymphopenia, and increased d-dimers. Imaging studies revealed multifocal airspace disease and ground-glass opacities. SARS-CoV-2 PCR and serologies were negative. All patients reported a history of vaping, prompting E-cigarette, or vaping, product use-associated lung injury (EVALI) diagnosis. MIS-C has overlapping clinical and laboratory features highlighting the added challenge of diagnosing EVALI during the COVID-19 pandemic.
\end{abstract}

EVALI versus MIS-C, one more overlapping diagnosis to consider

Diego A Cruz-Vidal, $\mathrm{MD}^{1}$, Eric S Mull, DO ${ }^{2}$, Jeanette Taveras, $\mathrm{DO}^{1}$, Richard Shell, $\mathrm{MD}^{2}$, Garrett W. Hunt, $\mathrm{MD}^{1}$, Brain Fowler, BA ${ }^{1}$, Rebecca Wallihan, $\mathrm{MD}^{1}$, Guliz Erdem, $\mathrm{MD}^{1}$

Affiliations : ${ }^{1}$ Division of Infectious Diseases, Nationwide Children's Hospital, Columbus, Ohio, ${ }^{2}$ Division of Pulmonary Medicine, Nationwide Children's Hospital, Columbus, Ohio

Address correspondence to : Guliz Erdem, Division of Infectious Diseases, Nationwide Children's Hospital, 700 Children's drive, Columbus, OH, 43205, [Guliz.Erdem@nationwidechildrens.org], 614-722-8925

Short title: EVALI versus MIS-C

Conflict of Interest Disclosures: The authors have no conflicts of interest relevant to this article to disclose

Funding/Support : No funding was secured for this study.

\section{Abbreviations :}

MIS-C: multisystem inflammatory syndrome in children; EVALI: E-cigarette, or vaping, product useassociated lung injury; COVID-19: Coronavirus disease 2019; CDC: Centers for Disease Prevention and Control; ESR: Erythrocyte sedimentation rate; CRP: C-reactive protein; PCT: Procalcitonin; LDH: Lactate Dehydrogenase; NP: Nasopharyngeal; FRVP: FilmArrayß respiratory panel; THC: tetrahydrocannabinol; CT: Computerized tomography; BAL: Bronchoalveolar Lavage

\section{Summary :}

We describe 6 teenagers with suspected MIS-C in which EVALI was the final diagnosis; we emphasis the importance of including EVALI as a differential diagnosis.

\section{Contributors' Statement:}


Dr. Cruz was the infectious disease clinical fellow who evaluated the patients and he was the primary author of this case report. Dr. Mull, Dr. Taveras followed the patients as pulmonary medicine and infectious disease fellows. Dr. Hunt and Mr. Fowler treated some of the patients. Dr. Shell was the precepting physician for Dr. Mull and followed some of the patients. Dr Erdem was the infectious disease attending who managed and consulted the patients. She supervised the writing and editions of this manuscript. All authors noticed the overlapping presentations of MIS-C and EVALI and came with the conception. In addition, all authors approved the final manuscript as submitted and agree to be accountable for all aspects of the work. All authors made substantial contribution(s) to conception, acquisition of patient data, or analysis and interpretation of patient characteristics. All authors participated in drafting the manuscript; and final approval of the version to be published; and they are all in agreement to be accountable for all aspects of the work in ensuring that questions related to the accuracy or integrity of any part of the work are appropriately investigated and resolved.

\begin{abstract}
We describe six teenagers presenting with fever and severe abdominal symptoms admitted with concerns for multisystem inflammatory syndrome in children (MIS-C). Laboratory evaluation revealed elevated markers of inflammation, lymphopenia, and increased d-dimers. Imaging studies revealed multifocal airspace disease and ground-glass opacities. SARS-CoV-2 PCR and serologies were negative. All patients reported a history of vaping, prompting E-cigarette, or vaping, product use-associated lung injury (EVALI) diagnosis. MIS-C has overlapping clinical and laboratory features highlighting the added challenge of diagnosing EVALI during the COVID-19 pandemic.
\end{abstract}

\title{
Introduction
}

Coronavirus disease 2019 (COVID-19) was thought to largely spare children until clinicians in the United Kingdom reported a cluster of children with fever and hyperinflammation in late April $2020^{1}$. Similar reports from Italy, France, and the United States led the Centers for Disease Prevention and Control (CDC) to define this illness as Multisystem Inflammatory Syndrome in children (MIS-C) (CDC). The case definition of MIS-C includes the presence of fever, laboratory evidence of inflammation, multisystem organ involvement, and evidence of SARS-CoV-2 infection or an epidemiologic link to a person with COVID- $19^{2}$. The broad case definition and wide spectrum of clinical presentations, observed with MIS-C, coupled with lack of pathognomonic signs and diagnostic tests have posed new challenges in the evaluation of children presenting with fever and elevated inflammatory markers due to the risk of serious and life-threatening illnesses that must be considered in these patients.

In this report, we describe six patients admitted to the hospital with concerns for MIS-C who were later diagnosed with EVALI.

\section{Case reports (Table 1)}

\section{Case 1}

A 16-year-old white male presented with 4 days of fevers to $38.7^{\circ} \mathrm{C}\left(101.4^{\circ} \mathrm{F}\right)$, nausea, severe abdominal pain, diarrhea, cough, and dyspnea. He denied any known exposure to COVID-19 infected individuals. He was admitted due to his tachypnea and concerns of MIS-C. Shortly after admission, he developed worsening tachypnea and hypoxia, along with difficulty breathing on nasal cannula requiring transitioning to high flow nasal cannula oxygen on second day of admission. Laboratory workup was remarkable for lymphopenia, elevated erythrocyte sedimentation rate (ESR), C-reactive protein (CRP), procalcitonin (PCT), ferritin, and lactate dehydrogenase (LDH). Chest X-Ray showed bibasilar interstitial infiltrates. Chest computed tomography (CT) revealed bilateral multifocal ground-glass opacities (Figure 1). Nasopharyngeal (NP) SARS-CoV-2 PCR and serum antibodies were negative twice. FilmArrayß respiratory panel (FRVP) (Biofire Defense, Salt Lake City, Utah) was negative. He remained febrile, with ongoing respiratory distress despite empiric therapy with ceftriaxone and azithromycin. He later disclosed vaping every week with products obtained from his friends. Urine drug screen was positive for tetrahydrocannabinol (THC). Intravenous steroids were 
started due to suspected EVALI. He was weaned to room air within 48 hours, his infiltrates improved on repeat chest X-ray. He was discharged after 8 days of hospital stay.

\section{Case 2}

A 16-year-old white female presented with 6 days of fevers to $39.2^{\circ}\left(102.7^{\circ} \mathrm{F}\right)$, severe abdominal pain, and vomiting. She had tachypnea and a transient desaturation to $88 \%$ before admission. Her ESR and CRP were significantly elevated. A CT of the abdomen showed no abdominal abnormalities however bilateral basilar lung opacities were noted. A dedicated CT of the chest showed multifocal mixed ground glass and consolidative airspace disease involving the bilateral upper and lower lobes. SARS-CoV-2 NP PCR and serology were negative. Additional infectious work-up included negative FRVP, fungal antibody panel, histoplasma urine and blood antigens and legionella urine antigen. She was empirically started on levofloxacin. She reported a history of vaping over a year ago. Family reported that she may have been vaping more recently. She was discharged as her clinical condition improved. No relapsing disease was noted on her follow-up 2 weeks after discharge and her chest X-ray findings resolved.

\section{Case 3}

An 18-year-old white male presented with 1 week of persistent tactile fevers, chest pain, shortness of breath, vomiting, and diarrhea after two days of outpatient therapy with azithromycin and levofloxacin. Upon admission, ceftriaxone was started, and levofloxacin was continued. Initial laboratory work-up showed lymphopenia, elevated CRP, PCT, and LDH. His FRVP and SARS-CoV-2 PCR were negative. Fungal antibody panel, blood and urine histoplasma antigens and urine Legionella antigen were negative. He reported frequent use of marijuana and vaping. A CT of the chest showed multifocal airspace disease with relative subpleural sparing. A bronchoalveolar lavage (BAL) was performed with negative cultures for bacterial, mycobacterial, and fungal etiologies. BALPneumocystis jirovecii PCR was positive with negative stains or signs of eosinophilic pneumonia on cytology. He clinically improved with improved inflammatory markers without the need for supplemental oxygen. He was discharged after 3 days.

\section{Case 4}

A 16-year-old white male presented with 6 days of fever to $39.2^{\circ} \mathrm{C}\left(102.6^{\circ} \mathrm{F}\right)$, cough, shortness of breath, vomiting, and diarrhea. He was prescribed albuterol, azithromycin, and prednisone prior to admission, but was unable to adhere to treatment due to vomiting. On admission, he had lymphopenia; elevated CRP, PCT, and ESR. His SARS-CoV-2 PCR testing was negative. His FRVP, anti-streptolysin O, fungal antibody panel, histoplasma serum and urine antigens, QuantiFERON TB Gold plus, and sputum fungal and mycobacterial cultures were negative. A chest CT showed multifocal ground-glass opacities bilaterally. He was empirically started on ceftriaxone and azithromycin. He required supplemental oxygen until day 6 of admission. He initially acknowledged marijuana usage but denied vaping, later clarifying usage of E-cigarettes. He was started on steroids and after significant clinical improvement was discharged home on day 8 of hospitalization. He remained asymptomatic during the outpatient follow up.

\section{Case 5}

A 17-year-old white male presented with 7 days of cough and 4 days of fever to $39.4^{\circ} \mathrm{C}\left(103^{\circ} \mathrm{F}\right)$, myalgias, and fatigue with emesis. On admission he had mild respiratory distress requiring nasal cannula oxygen. A chest X-ray showed bilateral lung opacities. Azithromycin and ceftriaxone were empirically started. His initial laboratory workup was remarkable for leukocytosis, elevated ESR, CRP, and LDH. SARS-CoV-2 PCR was obtained twice and negative. in both opportunities, His FRVP and SARS-CoV-2 antibodies were negative. Legionella urine antigen, M. pneumoniae throat PCR, histoplasma urine and blood antigens, EBV profile, HIV ELISA, and QuantiFERON TB-gold plus were negative. Later he acknowledged E-cigarette and marijuana usage. A THC urine test that was positive. CT of the chest showed diffuse bilateral ground-glass opacities, with regions of superimposed interlobular septal thickening. Antibiotic treatment was discontinued. He was started on steroids with significant improvement in 24 hours and was subsequently discharged.

\section{Case 6}


A 17 -year-old white male presented with 6 days of fever to $39.4^{\circ} \mathrm{C}\left(103^{\circ} \mathrm{F}\right)$, abdominal pain and vomiting. At an outside facility a CT of the abdomen was done showing no abdominal abnormalities however bilateral basilar lung opacities were noted. At admission was febrile with abdominal pain, had significantly elevated inflammatory markers. His FRVP, SARS-CoV-2 PCR and antibodies were negative. He acknowledged the use of E-cigarettes. His THC-urine test was positive. A dedicated CT of the chest showed bilateral ground-glass opacities with subpleural sparing and the infectious workup was negative, especially for histoplasma infection that was high on the differential. He improved after 5 days of hospital stay and throughout infectious testing, without any specific treatment, for which EVALI was diagnosed and follow-up as an outpatient was arranged.

\section{Discussion}

Pediatric SARS-CoV-2 infection can be complicated by a potentially life-threatening hyperinflammatory condition termed MIS-C. The severity of MIS-C, coupled with a variety of non-specific but common symptoms, such as fever and abdominal pain, as well as the broad case definition, create challenges for health care providers. Although, the presence of positive SARS-CoV-2 PCR and/or serology is helpful for diagnosis, testing may not be readily available and turnaround time can be delayed. Additionally, many children currently have potential recent exposures to COVID-19. MIS-C symptoms and clinical manifestations may overlap with those of some infectious respiratory diseases, including COVID-19, toxic shock syndrome, macrophage activation syndrome, and Kawasaki syndrome ${ }^{3}$.

MIS-C, much like EVALI, may cause severe respiratory symptoms, constitutional complaints, and similar chest imaging findings ${ }^{3}$. Our patients were admitted with fever or history of fever, severe abdominal pain, lymphopenia, significantly elevated inflammatory markers in all, and ground-glass opacities of lungs suggestive of MIS-C. D-dimers were also elevated. Differing from reported MIS-C patients was that our EVALI patients were of white ethnicity ${ }^{4,5}$. Although our center is fortunate to have SARS-CoV-2 PCR and serology results available to us in a short period of time, these patients still had possible COVID-19 exposure; creating further challenges for treatment and management decisions. Thorough history taking helped to differentiate the illnesses and avoid unwarranted treatments in our patients. EVALI is an additional overlapping diagnosis that can be considered in older children presenting for evaluation of MIS-C, especially in the setting of negative SARS-CoV-2 testing and vaping history.

Similar to our cases, a significant number of EVALI patients reported sharing the same device or product with friends and family members, increasing their risk for COVID-19 infection ${ }^{6}$. EVALI patients also reported vaping more to cope with pandemic associated stressors and anxiety ${ }^{3}$. These factors may partially explain the matching geographic distribution of hospitalized EVALI cases and reported COVID-19 cases currently in the US ${ }^{7,8}$. We believe these findings have direct implications for health care providers when MIS-C is considered in older children as EVALI could present similarly. A comprehensive e-cigarette use history helped our patients have a prompt diagnosis and treatment.

\section{Conclusions}

MIS-C has overlapping clinical and laboratory features highlighting the added difficult challenge of diagnosing EVALI during the COVID-19 pandemic. We stress the importance of a through history and possible drug screening when COVID-19 testing is negative in concerning clinical presentations.

\section{References}

1. Riphagen S, Gomez X, Gonzalez-Martinez C, Wilkinson N, Theocharis P. Hyperinflammatory shock in children during COVID-19 pandemic. Lancet 2020;395:1607-1608.

2. Centers for Disease Control and Prevention. Multisystemic Inflammatory Syndrome in Children (MIS-C) associated with Coronavirus Disease 2019 (COVID-19). CDC Health Alert Network. https://emergency.cdc.gov/han/2020/han00432.asp. Published 2020. Accessed November 11, 2020.

3. Callahan SJ, Harris D, Collingridge DS, Guidry DW, Dean NC, Lanspa MJ, Blagev DP. Diagnosing EVALI in the Time of COVID-19. Chest 2020. 
4. Krishnasamy VP, Hallowell BD, Ko JY, Board A, Hartnett KP, Salvatore PP, Danielson M, Kite-Powell A, Twentyman E, Kim L, et al. Update: Characteristics of a Nationwide Outbreak of E-cigarette, or Vaping, Product Use-Associated Lung Injury - United States, August 2019-January 2020. MMWR Morb Mortal Wkly Rep 2020;69:90-94.

5. Godfred-Cato S, Bryant B, Leung J, Oster ME, Conklin L, Abrams J, Roguski K, Wallace B, Prezzato E, Koumans EH, et al. COVID-19-Associated Multisystem Inflammatory Syndrome in Children - United States, March-July 2020. MMWR Morb Mortal Wkly Rep 2020;69:1074-1080.

6. Gaiha SM, Cheng J, Halpern-Felsher B. Association Between Youth Smoking, Electronic Cigarette Use, and Coronavirus Disease 2019. J Adolesc Health 2020;67:519-523.

7. Centers for Disease Control and Prevention. CDC COVID Data Tracker. https://covid.cdc.gov/coviddata-tracker/\#cases_totalcases. Published 2020. Accessed November 11, 2020.

8. Centers for Disease Control and Prevention. Outbreak of Lung Injury Associated with the Use of ECigarette, or Vaping, Products. https://www.cdc.gov/tobacco/basic_information/e-cigarettes/severe-lungdisease.html. Published 2020. Accessed November 11, 2020.

Table 1. Demographic, clinical and laboratory findings of patients.

Figure 1. CT of the chest from Patient 1. Diffuse hazy ground-glass opacification seen throughout both lungs.

\section{Hosted file}

evalitablenovember9 (1).pdf available at https://authorea.com/users/375287/articles/492581evali-versus-mis-c-one-more-overlapping-diagnosis-to-consider

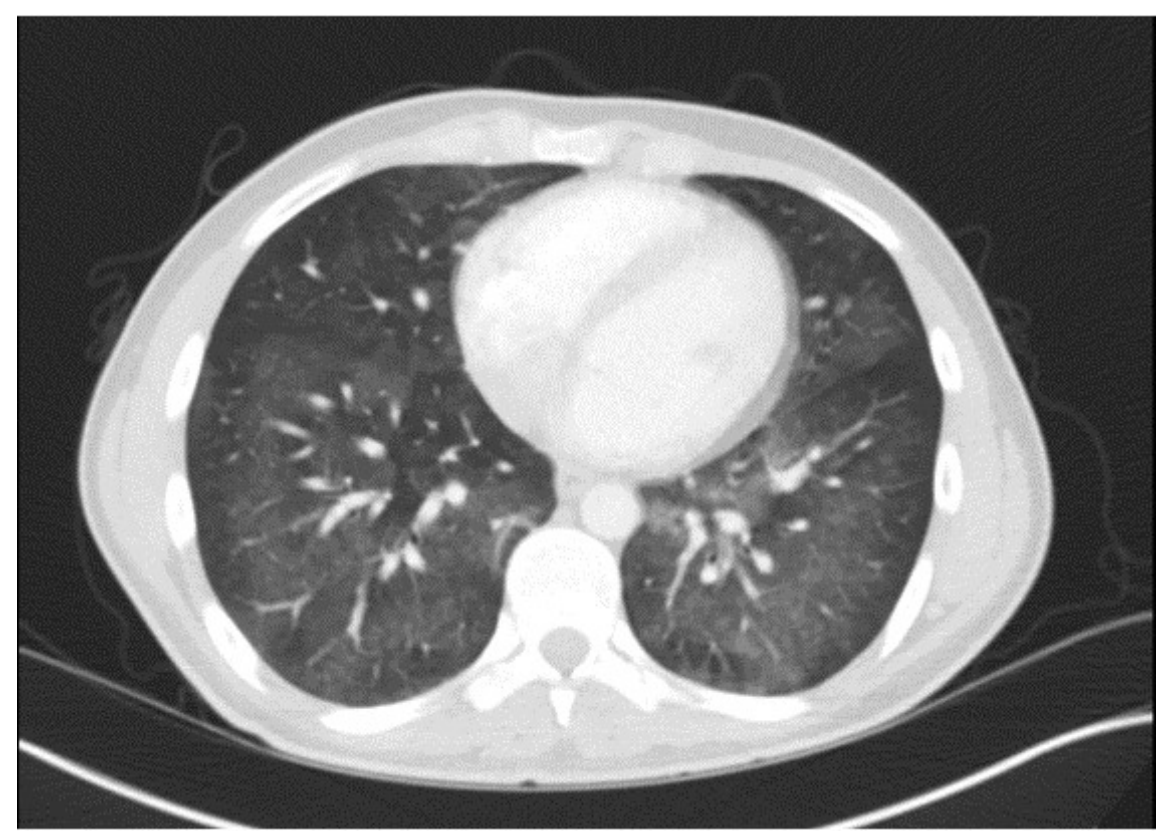

